Please quote as: Wulf, J.; Blohm, I.; Brenner, W. \& Leimeister, J. M. (2014): Massive Open Online Courses . In: WIRTSCHAFTSINFORMATIK, Erscheinungsjahr/Year: 2014. Seiten/Pages: Online First. 


\section{Massive Open Online Courses}

\section{DOI 10.1007/s11576-014-0405-7}

\author{
Die Autoren \\ Dr. Jochen Wulf $(\varangle)$ \\ Dr. Ivo Blohm \\ Prof. Dr. Walter Brenner \\ IWI-HSG \\ Universität St. Gallen \\ Müller-Friedberg-Strasse 8 \\ 9000 St. Gallen \\ Schweiz \\ jochen.wulf@unisg.ch \\ ivo.blohm@unisg.ch \\ walter.brenner@unisg.ch
}

Prof. Dr. Jan Marco Leimeister Lehrstuhl für Wirtschaftsinformatik

Universität Kassel

Nora-Platiel-Straße 4

34127 Kassel

Deutschland

leimeister@uni-kassel.de

und

Institut für Wirtschaftsinformatik

Universität St. Gallen

Müller-Friedberg-Strasse 8

9000 St. Gallen

Schweiz

Eingegangen: 2013-07-26

Angenommen: 2013-12-03

Angenommen nach einer Überarbeitung durch Prof. Dr. Sinz.

This article is also available in English via http://www.springerlink.com and http://www.bise-journal.org: Jochen J, Blohm I, Leimeister JM, Brenner W (2014) Massive Open Online Courses. Bus Inf Syst Eng. doi: 10.1007/s12599-014-0313-9.

(c) Springer Fachmedien Wiesbaden 2014

\section{Massive Open Online Courses - Die Demokratisierung von Bildung?}

Das Georgia Institute of Technology (Georgia Tech) ist eine der weltweit führenden Universitäten in der Informatikausbildung. Ab dem Wintersemester 2014 bietet die Georgia Tech einen Master in „Computer Science“ an, bei dem die einzelnen Kurse ausschließlich als „Massive Open Online Course (MOOCs)" angeboten werden. ${ }^{1}$ Die Studiengebühren sollen, im Vergleich zum traditionellen Masterprogramm, nur einen Bruchteil betragen, um die Zugänglichkeit des Bildungsangebots zu vergrößern. Zur Umsetzung dieses Vorhabens kooperiert Georgia Tech mit dem Telekommunikationsunternehmen AT\&T und der privaten Bildungsorganisation Udacity. ${ }^{2}$ AT\&T unterstützt das Programm mit Finanzmitteln von zwei Millionen US-Dollar und nutzt das Programm zur Ausbildung der eigenen Mitarbeiter sowie zur Rekrutierung von Absolventen. Udacity hat durch das Angebot von MOOCs für eine Teilnehmerzahl von bis zu 300.000 Studenten globale mediale Aufmerksamkeit erreicht. Einige Udacity-MOOCs werden bereits von Universitäten in Freiburg, München und Berlin anerkannt. ${ }^{3}$ Angesichts dieser Entwicklung werden traditionelle Lehrformen und institutionelle Ansätze in der akademischen Bildung zunehmend in Frage gestellt (Vardi 2012).

Was sind MOOCs? MOOCs sind von Professoren oder anderen Experten durchgeführte internetbasierte Onlinekurse mit einer nichtlimitierten Anzahl von Teilnehmern. Es lassen sich folgende konstitutive Merkmale nennen (Clow 2013; McAuley et al. 2010; Vardi 2012):

- Große Teilnehmerzahl („massive“): Im Unterschied zu traditionellen Fernstudienkursen richten sich MOOCs an eine unbegrenzte Anzahl von Teilnehmern.

- Freie Zugänglichkeit („open“): Es bestehen keine oder nur sehr geringe formale Teilnahmevoraussetzungen. Die
Veranstaltungen richten sich an einen globalen Adressatenkreis. Fachspezifische Vorkenntnisse sind nur dann nachzuweisen, wenn der Kurs in einen Studienplan eingebettet ist. Zudem sind MOOCs oftmals gebührenfrei oder mit sehr geringen Teilnahmegebühren belegt.

- Digitalisierung („online“): Die Veranstaltung wird vollständig über das Internet durchgeführt, ist also nicht ortsgebunden. Die Digitalisierung umfasst die Bereitstellung und die Vermittlung der Lerninhalte, die soziale Interaktion der Teilnehmer und die Durchführung von Lernzielabfragen und Prüfungen.

- Didaktisches Konzept („course“): Lerninhalte werden einem lehrmethodischen Konzept folgend strukturiert. Die Wissensvermittlung und -erarbeitung ist an Lernzielen ausgerichtet. Beispielhafte Gestaltungselemente sind eine Zeitplanung, eine Vorstrukturierung der Lerninhalte, die Steuerung von sozialen Lerninteraktionen sowie die Durchführung von Lernzielabfragen und Prüfungen.

Aus dem Blickwinkel der Wirtschaftsinformatik stellen MOOCs ein innovatives, internetbasiertes Geschäftsmodell für die Finanzierung, Gestaltung und die Erbringung von Lehrdienstleistungen dar. Durch die zunehmende Digitalisierung und die damit einhergehende Strukturierung der Dienstleistungen unterliegen diese den Gesetzen der Internetökonomie (vgl. Shapiro und Varian 1999; Grenzkosten zusätzlicher Teilnehmer tendieren gegen Null, Auftreten von Netzwerk- und Long-Tail-Effekten), sodass universitäre Bildung und berufliche Weiterbildung einer breiten Masse zugänglich gemacht werden können. Dadurch ergeben sich große Potenziale (z. B. Effektivitäts- und Effizienzsteigerungen in der Lehre; Leimeister 2012, S. 46-102) und Herausforderungen (z. B. neue Wettbewerber) für betriebliche und universitäre Lehrdienstleister.

\footnotetext{
${ }^{1}$ http://www.omscs.gatech.edu/.

2 https://www.udacity.com

${ }^{3}$ http://blogs.faz.net/netzwirtschaft-blog/2013/06/07/online-akadamie-udacity-uni-abschlusse-werden-verschwinden-3495/.
} 
Tab. 1 Mechanismen und Technologien zur Digitalisierung und Skalierung von Lehrveranstaltungen

Peer-Support

Peer-Grading

Gamification

Learning-Analytics

Prüfungsüberwachung

Digitale

Rechteverwaltung
Zur Unterstützung der Auseinandersetzung mit den Lerninhalten kommt es zu einer Institutionalisierung von Peer-Support-Mechanismen, sodass die Teilnehmenden in die Lage versetzt werden, sich gegenseitig bei Fragen und Problemen zu helfen. Dafür werden z. B. Diskussionsforen verwendet (Clow 2013). Die aktive

Diskussionsteilnahme wird häufig als wesentlicher Bestandteil des Kurs- und Bewertungskonzeptes eingesetzt. Es gibt darüber hinaus viele weitere Möglichkeiten des IT-Einsatzes für kollaboratives Lernen (Haake et al. 2012).

Prüfungen und Tests werden im Rahmen von MOOCs häufig von Mitstudenten bewertet (Peer-Grading). Verglichen mit den Bewertungen des Lehrpersonals, kommt es hierbei in der Regel nur zu geringen Abweichungen (Sadlar und Good 2006). Zudem werden beim Peer-Grading Verfahren zur Kalibrierung der unterschiedlichen Bewertungen verschiedener Studenten eingesetzt (Robinson 2001).

Um Anreize für eine aktive Teilnahme an den Forumsdiskussionen und am Peer-Support zu schaffen, werden oftmals Gamification-Elemente, wie z. B. Badges (Abzeichen) oder eng miteinander verzahnte Aufgabenketten, eingesetzt. Darunter versteht man den Einsatz von Spiel-Design-Elementen in Produkten, Dienstleistungen und Informationssystemen, um deren Nutzung zu vergrößern (Blohm und Leimeister 2013). Zudem werden Badges neben klassischen Zertifikaten eingesetzt, um die erworbenen Kompetenzen eines Kursteilnehmers zu bestätigen. ${ }^{\mathrm{a}}$

Aufgrund der vollständigen Digitalisierung der Studenteninteraktionen, der Lernzielabfragen und der Prüfungsleistungserfassung können die Effektivität der eingesetzten Lehrmethoden gemessen und die Lerninhalte an das Leistungsniveau des individuellen Teilnehmers angepasst werden (Cooper und Sahami 2013; Sadlar und Good 2006).

Zur Durchführung von Prüfungen können informationstechnische Verfahren eingesetzt werden, um die Identität des Prüfungsteilnehmers zu überprüfen und die Prüfungsabnahme zu überwachen.

Parallel zum Kurs kann Teilnehmern Zugriff auf elektronische Fachbücher gewährt werden. Hierzu werden digitale Technologien zum Verleih von eBooks und zur Rechteverwaltung eingesetzt.

${ }^{a}$ http://www.forbes.com/sites/jamesmarshallcrotty/2013/02/25/new-improved-badges-give-credential-meat-to-mooc-revolution/

\section{Bisherige Forschung zu MOOCs}

Die gegenwärtige wissenschaftliche Diskussion um MOOCs fokussiert auf die unterschiedlichen Typen von MOOCs, die dabei eingesetzten didaktischen Konzepte sowie die Technologien und Mechanismen, die eine solche Skalierung von Lehrdienstleistungen ermöglichen.

Clow (2013) unterscheidet zwei grundsätzliche Ausprägungsformen von MOOCs: cMOOCs und xMOOCs. Die „connectivist MOOCs“ (cMOOCs) fußen auf den pädagogischen Prinzipien des Konnektivismus. Sie sind mit Seminaren vergleichbar, in denen Teilnehmer Inhalte auswerten bzw. strukturieren, neue Inhalte, wie z.B. Texte und Kommentare, verfassen und den anderen Teilnehmern zur Verfügung stellen. Der Konnektivismus sieht die Interaktion zwischen Teilnehmern als wesentliche Quelle der Wissensentstehung (Kop und Hill 2008).

xMOOCs hingegen sind durch die Digitalisierung von traditionellen Vorlesungsformaten entstanden und nutzen behavioristische Lehrmethoden (Clow 2013). xMOOCs bestehen in der Regel aus kurzen Videosequenzen zur Vermittlung der Lerninhalte und direkten Lernabfragen (Vardi 2012). Aufgrund der großen Masse an Teilnehmenden ist eine individuelle und direkte Interaktion mit den Lehrkräften nicht realisierbar. Aus diesem Grund bedienen sich xMOOCs unterschiedlicher Technologien zur Gewährleistung der Skalierbarkeit, z. B. einer automatisierten Bewertung von Multiple-Choice-Fragen, aus der Softwareentwicklung bekannten Testverfahren zur Überprüfung der Korrektheit von Programmcode (Cooper und Sahami 2013) oder Verfahren zur automatisierten Plagiatsprüfung. Tabelle 1 fasst wesentliche didaktische Mechanismen und Technologien zusammen, die bei xMOOCs verwendet werden.

Aufgrund der großen Teilnehmeranzahl und dem systematischen Einsatz dieser Technologien sind MOOCs von Ansätzen des integrierten Lernens („Blended Learning") abzugrenzen, in denen eLearning-Elemente und Präsenzveranstaltungen kombiniert werden. Im Vergleich zu klassischen, onlinebasierten Fernstudienangeboten ist keine individuelle Interaktion mit dem Lehrkörper vorgesehen. Auch ist eine Abgrenzung zu anderen internetbasierten Formaten der Wissensvermittlung, wie z. B. „Webinaren", vorzunehmen, denen es häufig an einer vergleichbaren Interaktivität und einer lehrmethodischen Untermauerung fehlt.

\section{Geschäftsmodelle zur Durchführung von MOOCs}

Aus Perspektive der Wirtschaftsinformatik stellen MOOCs eine Geschäftsmodellinnovation für Lehrdienstleistungen dar, die in zunehmender Weise in digitalen Wertschöpfungsnetzwerken erbracht werden. Vereinfachend lassen sich dabei drei grundsätzliche $\mathrm{Ge}$ schäftsmodelle zur Bereitstellung von MOOCs identifizieren (siehe Abb. 1). Zur Abgrenzung werden die folgenden Funktionen unterschieden, die abhängig vom Geschäftsmodell von verschiedenen Akteuren (z. B. Universitäten, Unternehmen, IT-Service-Providern, Veranstaltungsteilnehmern, Drittinstitutionen) wahrgenommen werden können:

- Finanzierung: Bereitstellung der Mittel zur Finanzierung der Veranstaltungsdurchführung und der technischen Plattform

- Lehrplanung und -betrieb: Didaktische Planung, Aufbereitung und Bereitstellung der Lerninhalte, soziale Lehrinteraktionen und Leistungsbewertung

- Plattformbetrieb und Vermarktung: Entwicklung und Betrieb der technischen Systeme zur Distribution der Lerninhalte, zur Unterstützung des 


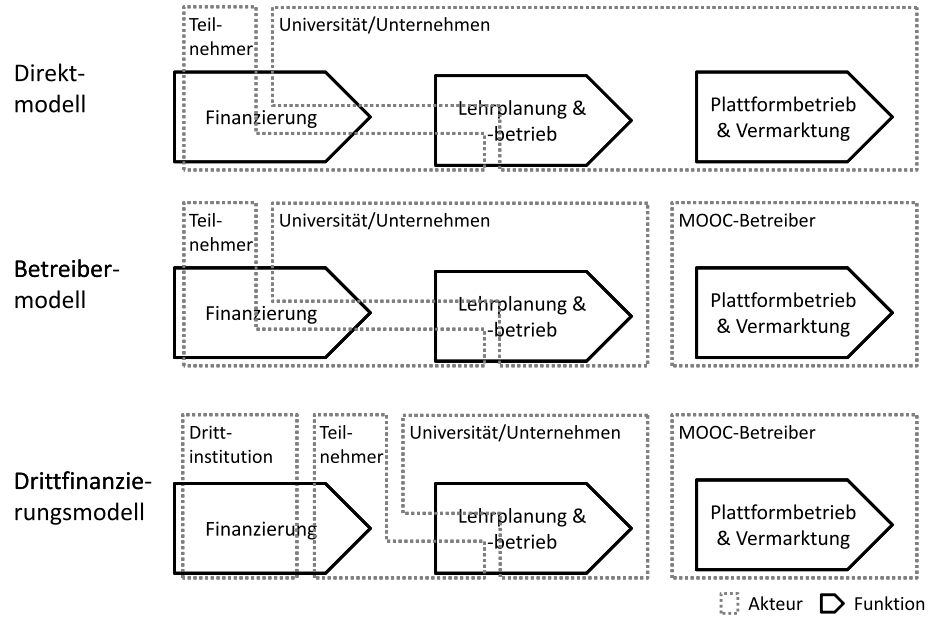

Abb. 1 MOOC Geschäftsmodelle

Lehrbetriebs, zum Nutzermanagement und zur Vermarktung

Im Direktmodell werden die Veranstaltung und die technische Plattform von ein und derselben Institution verantwortet. Finanziert wird dieses Modell durch Gebühren zur Ausstellung des Abschlusszertifikates oder durch die Ausrichter der Lehrveranstaltung, die die Veranstaltung zur Vermarktung komplementärer Angebote nutzen. Dieses Modell wird in erster Linie von Unternehmen eingesetzt, um Weiterbildungsangebote für eigene Mitarbeiter oder Kunden zu entwickeln. Teilweise wird dieses Modell aber auch von klassischen Bildungseinrichtungen verfolgt, um die Reichweite des eigenen Lehrangebots zu vergrößern. Beispiele für dieses Modell sind openH$\mathrm{PI}^{4}$ des Hasso-Plattner-Instituts oder das openSAP-Programm ${ }^{5}$ des Unternehmens SAP.

Im Betreibermodell ist ein dedizierter Betreiber für die technische Infrastruktur verantwortlich. Dieser Betreiber stellt MOOCs verschiedener Lehrinstitutionen bereit und übernimmt auch die Vermarktung der Lehrveranstaltungen sowie das Nutzermanagement. Eventuelle Einnahmen aus der Ausstellung von Zertifikaten werden zwischen dem Ausrichter der Lehrveranstaltung und dem Plattformbetreiber geteilt. Beispiele für dieses Modell sind MOOC2 degree ${ }^{6}$ und Udemy. ${ }^{7}$
Im Drittfinanzierungsmodell werden MOOCs nicht durch die Teilnehmenden oder die Ausrichter finanziert, sondern durch Erlöse aus Angeboten für Drittinstitutionen. So verdienen z. B. Plattformbetreiber durch die Vermittlung von Teilnehmerinformationen an potenzielle Arbeitgeber oder Werbetreibende. Werden im Sinne eines Crowdsourcings reale Problemstellungen von Firmen im Rahmen von MOOCs durch Kursteilnehmer bearbeitet, entsteht eine weitere Finanzierungsquelle. ${ }^{8}$ Zudem können Lizenzen für MOOCs an andere Universitäten vertrieben werden, die diese in ihr Curriculum aufnehmen und integrierte Lehrveranstaltungen anbieten können. Beispielsweise nutzen die Plattformbetreiber Udacity und Coursera ${ }^{9}$ derartige Einkommensquellen.

\section{Potenziale, Herausforderungen und Ausblick}

Aufgrund der Digitalisierung und dem Entstehen digitaler Wertschöpfungsnetzwerke bieten MOOCs für Bildungsinstitutionen und Unternehmen eine Vielzahl von Potenzialen zur Entwicklung innovativer Lehrangebote sowie für deren effektive und effizientere Erbringung:

- Lernendenintegration: Mit dem systematischen Einsatz von Interaktionsmechanismen, wie z. B. Peer-
Support und Peer-Grading, werden traditionell von Lehrdienstleistern erbrachte Wertschöpfungsschritte systematisch an MOOC-Teilnehmer ausgelagert. Aus einer ökonomischen Perspektive tragen Kursteilnehmer damit aktiv zur Kostenreduktion der Lehrangebote bei, indem sie Teilaufgaben des Lehrprozesses, insbesondere in den Bereichen der Betreuung und Bewertung, übernehmen. Aus einer didaktischen Perspektive entspricht dies einer Vertiefung und Absicherung von Lerninhalten, da interaktive Wertschöpfung in der Regel mit großen Lerneffekten bei den Teilnehmenden einhergeht (Reichwald und Piller 2009).

- Marginale Grenzkosten: Aufgrund der vollständigen Digitalisierung der Lehrdienstleistungen, der hohen Automatisierung der Lehrinteraktionen und des systematischen Ausnutzens der Möglichkeiten interaktiver Wertschöpfung sind die Grenzkosten der Skalierung von Lehrveranstaltungen marginal. Eine mögliche Konsequenz sind dramatische Kostenreduktionen in der Ausund Weiterbildung (Vardi 2012).

- Long-Tail-Angebote: Die Orts- und Zeitungebundenheit von MOOCs ermöglicht die Adressierung eines globalen Teilnehmerkreises, insbesondere von Berufstätigen. Hierdurch können auch spezialisierte Kurse wirtschaftlich angeboten werden.

- Individualisierung von Lehrdienstleistungen: Learning-Analytics ermöglicht eine Anpassung der Lerninhalte und der eingesetzten Lehrmethoden an den einzelnen Teilnehmer (Cooper und Sahami 2013). Der Einsatz vorhandener Instrumente des eLearning und von MOOC-Technologien kann sich an individuellen Lernzielen und Lernleistungen orientieren, sodass ohne großen Mehraufwand unterschiedliche Versionen der gleichen Lehrveranstaltung angeboten werden können. Ansätze des Service-Design im eLearning können hier unterstützend eingesetzt werden (Wegener et al. 2012).

- Netzwerkeffekte: Die vermehrte Etablierung von MOOCs könnte eine

\footnotetext{
${ }^{4}$ https://openhpi.de/?locale $=$ de.

${ }^{5}$ https://open.sap.com/.

${ }^{6}$ http://www.mooc2degree.com/.

${ }^{7}$ https://www.udemy.com/.

${ }^{8}$ http://trust.guidestar.org/2013/04/25/connect-with-students-to-mooc-source-your-data/.

${ }^{9}$ https://www.coursera.org/.
} 
deutlich höhere Konzentration des Bildungsmarktes zur Folge haben, sodass Reputation und Marke von Universitäten im Wettbewerb um Teilnehmer eine immer größere Bedeutung zukommt. ${ }^{10}$ So könnten auch im Bildungsbereich verstärkt Mechanismen der Aufmerksamkeitsökonomie zur Geltung kommen und zur Ausbildung von Winner-Takes-All-Märkten führen. Dies ist insbesondere im Rahmen von Drittfinanzierungsmodellen von Bedeutung, bei denen MOOCAnbieter neue Erlösquellen erschließen. Werden zum Beispiel im Rahmen von Freemium-Angeboten Informationen über die MOOC-Teilnehmer wirtschaftlich erschlossen, so wächst das Erlösvolumen mit der Anzahl der Teilnehmer.

In MOOCs müssen Lernende viel Verantwortung für sich selbst übernehmen, da der Kontakt zu Lehrpersonen stark eingeschränkt ist. Sie müssen sicher mit den zur Verfügung gestellten Werkzeugen umgehen, ihre Zeit sinnvoll einteilen und sich selbst immer wieder zur Teilnahme motivieren. Dies stellt insgesamt hohe Anforderungen an die Lernenden (Kop 2011), die nicht alle Teilnehmenden erfüllen können. Entsprechend ist die Gefahr groß, dass viele Lernende einen Kurs nicht erfolgreich abschließen werden. Beispielsweise lag die Abbruchquote des MOOCs „Artificial Intelligence" an der Stanford University im Jahr 2011 bei rund $87 \%{ }^{11}$ Jedoch existiert diese Problematik in klassischen Präsenzveranstaltungen gleichermaßen. Auch in klassischen Kursen nehmen Interaktion, Lernzufriedenheit und Lernerfolg bei steigenden Teilnehmerzahlen ab (Cuseo 2007). Zudem zeigt sich in Community-basierten Online-Kursen, dass gerade Aspekte wie eine individuelle Unterstützung durch den Lehrenden, strukturierte Kleingruppenaufgaben und eine Bewertung von Einzel- und Gruppenleistungen wichtig für den Lernerfolg sind (Wegener und Leimeister 2012). Dies sind jedoch didaktische Maßnahmen, die in MOOCs aufgrund der hohen Teilnehmerzahlen schwieriger oder gar nicht zu realisieren sind. Die Automatisierung der Leistungsüberprüfung sowie Peer-Grading-Verfahren mögen hier durchaus helfen. Allerdings existieren gegenwärtig noch technische und rechtliche Schwierigkeiten. Hängt die Ausstellung eines Zertifikats bzw. die Benotung sehr stark vom Peer-Grading ab, ist dies aus juristischer Sicht fragwürdig. Automatisierte Tests wiederum lassen sich bei dem aktuellen Stand der Technik noch nicht für alle Lerninhalte und Lernziele anwenden. Man denke hier an Soft Skills wie rhetorische Fähigkeiten, die über einen Multiple-Choice-Test nicht praktikabel prüfbar sind.

Es ist fraglich, in welchem Maße MOOCs kompetitiv zu klassischen Bildungsangeboten von Universitäten, Hochschulen und Unternehmen einzustufen sind. Gegen signifikante Wettbewerbseffekte spricht die hohe Bedeutung der sozialen Interaktion mit dem Lehrkörper. Erste Lehrerfahrungen zeigen vielmehr, dass ein komplementärer Einsatz von MOOCs im Rahmen von integrierten Lehrkonzepten einen stärkeren Fokus auf interaktive Lehrelemente in Präsenzveranstaltungen erlaubt („Flipped Classroom“; Martin 2012).

\section{Literatur}

Blohm I, Leimeister JM (2013) Gamification - Gestaltung IT-basierter Zusatzdienstleistungen zur Motivationsunterstützung und Verhaltensänderung. WIRTSCHAFTSINFORMATIK 55(4):275-278
Clow D (2013) MOOCs and the funnel of participation. In: Proc 3rd international conference on learning analytics and knowledge (LAK'13), New York, S 185-189

Cooper S, Sahami M (2013) Reflections on Stanford's MOOCs. Communications of the ACM 56(2):28-30

Cuseo J (2007) The empirical case against large class size: adverse effects on the teaching, learning, and retention of firstyear students. Journal of Faculty Development 21(1):5-21

Haake J, Schwabe G, Wessner M (Hrsg) (2012) CSCL-Kompendium 2.0: Lehr- und Handbuch zum computerunterstützten kooperativen Lernen, 2. Aufl. Oldenbourg, München

Kop R (2011) The challenges to connectivist learning on open online networks: learning experiences during a massive open online course. The International Review of Research in Open and Distance Learning 12(3):18-38

Kop R, Hill A (2008) Connectivism: learning theory of the future or vestige of the past? IRRODL 9(3)

Leimeister JM (2012) Dienstleistungsengineering und -management. Springer, Heidelberg

Martin F (2012) Will massive open online courses change how we teach? Communications of the ACM 55(8):26-28

McAuley A, Stewart B, Siemens G, Cormier D (2010) The MOOC model for digital practice. http://www.elearnspace.org/ Articles/MOOC Final.pdf. Abruf am 201307-05

Reichwald R, Piller F (2009) Interaktive Wertschöpfung. Gabler, Wiesbaden

Robinson R (2001) Calibrated Peer Review ${ }^{\mathrm{TM}}$ an application to increase student reading \& writing skills. American Biology Teacher 63(7):474-480

Sadlar P, Good E (2006) The impact of self- and peer-grading on student learning. Educational Assessment 11(1):1-31

Shapiro C, Varian HR (1999) Information rules: a strategic guide to the network economy. Harvard Business School Press, Boston

Vardi MY (2012) Will MOOCs destroy academia? Communications of the ACM 55(11):5

Wegener R, Leimeister JM (2012) Virtual learning communities: success factors and challenges. International Journal of Technology Enhanced Learning 4(5/6):383-397

Wegener $R$, Menschner $P$, Leimeister JM (2012) Design and evaluation of a didactical service blueprinting method for large scale lectures. In: Proc international conference on information systems (ICIS), Orlando

\footnotetext{
${ }^{10}$ Sebastian Thrun (CEO Udacity) geht davon aus, dass es in 50 Jahren nur noch 10 Universitäten weltweit geben wird. http://www.economist.com/ news/international/21568738-online-courses-are-transforming-higher-education-creating-new-opportunities-best.

${ }^{11} \mathrm{http}: / /$ www.forbes.com/sites/collegeprose/2013/01/28/moocs-a-college-education-online/.
} 